Alfaro Mateu, V., Bastias Manresa, J. \& Salinas Hernández, F.J. (2016). Relación entre inteligencia emocional y notas de las áreas instrumentales en un grupo de tercero de primaria. Revista Electrónica Interuniversitaria de Formación del Profesorado, 19(3), 149-155.

\title{
Relación entre inteligencia emocional y notas de las áreas instrumentales en un grupo de tercero de primaria
}

\author{
Vicent Alfaro Mateu, Joaquin Bastias Manresa y Fco. José Salinas Hernández \\ (Universidad de Alicante, Alicante, España)
}

\section{Resumen}

La finalidad de este trabajo es conocer si existe relación entre las áreas instrumentales, lengua y matemáticas, y la Inteligencia emocional de un grupo de alumnos de tercero de primaria de un centro concreto. Participaron 26 alumnos a los cuales se aplicó el instrumento test EQ-i YV de BarOn adaptado para los alumnos. Las técnicas de análisis de correlación a través del SPSS permitirán comprobar si existe o no correlación entre la Inteligencia Emocional de los alumnos, los distintos aspectos de la misma y las áreas instrumentales.

En los resultados encontrados no existe correlación entre matemáticas y lengua castellana y el total del cuestionario. La correlación entre las áreas instrumentales, lengua castellana y matemáticas es altamente significativa. Del mismo modo, el total de resultados del cuestionario "E" correlaciona positivamente con las partes del cuestionario "A", "B", "C" $y$ "D", remarcando que hay una alta correlación entre el factor intrapersonal ("B") y el factor de adaptabilidad ("D"). En conclusión, no existe una correlación directa entre las notas de los estudiantes y los resultados del test de inteligencia emocional.

Palabras clave

Inteligencia emocional; rendimiento académico; Educación Primaria. 


\title{
Relation between emotional intelligence and instrumental areas grades in one group of third course of Primary Education
}

\begin{abstract}
The purpose of this research is to know if there is correlation between instrumental areas, Spanish language and Mathematics, and Emotional Intelligence in a group of third grade students of a particular school. BarOn's Test EQ-i YV was adapted and applied for twenty-six students who took part in it. The correlation analysis techniques through SPSS program will make possible to verify whether or not there is correlation between student's emotional intelligence, the different aspects of it and instrumental areas.

The results found no correlation between Maths and Spanish language and the total of the questionnaire "E". The correlation between the instrumental areas, Spanish Language and Mathematics is highly significant. In the same way, the total outcomes of questionnaire "E " correlates positively with partial questionnaire "A", "B", "C" and " $D$ " highlighting that there is a high correlation between intrapersonal factor (" $B$ ") and adaptability factor ("D"). In conclusion, a direct correlation between the students' marks and the results of the Emotional Intelligence Test does not exist.
\end{abstract}

\section{Keywords}

Emotional intelligence; academic performance; Primary Education

\section{Introducción}

La Inteligencia emocional, siempre ha tenido una compleja conceptualización debido a los numerosos aspectos a los que hace referencia. Considero importante revisar la evolución conceptual desde diferentes ámbitos y autores para determinar en que términos estamos trabajando. Miguel Luis Martín Jorge, Juan Antonio Mora Mérida, "Relación entre Inteligencia Emocional y personalidad en sus distintas concepciones teóricas", dentro del libro "Avances en el estudio de la Inteligencia Emocional: I Congreso Internacional de la Inteligencia Emocional”. Se atribuye como precursores de este término, al psicólogo Edward Thorndike (1920), quien consideró que existía la inteligencia social junto con la abstracta, referida al manejo de ideas, y la mecánica, referida a habilidad para entender y manejar objetos.

En 1983, Howard Gardner desarrolla su teoría de las Inteligencias Múltiples, que supuso un gran avance en cuanto a desvincular el Coeficiente Intelectual como medida de la inteligencia. Howard, introdujo 7 tipos de inteligencia, 2 de las cuales muy relacionadas con la competencia social, inteligencia interpersonal e intrapersonal, y que en la actualidad es una posible definición de la Inteligencia Emocional.

En 1990, los psicólogos Peter Salovey y John Mayer acuñaron este termino, aunque su auge se debe principalmente al libro que en 1995 publicó Daniel Goleman "Inteligencia emocional". En un excesivo resumen del libro, podemos considerar que Goleman, considera que necesitamos revisar la conceptualización de la Inteligencia, como un aspecto puramente cognitivo, Natalio Extremera Pacheco, Pablo Fernandez-Berrocal,(2004).

Mayer \& Salovey definen la inteligencia emocional como "la capacidad para percibir, valorar y expresar las emociones con exactitud; la capacidad para acceder y generar 
sentimientos que faciliten el pensamiento; la capacidad para entender la emoción y el conocimiento emocional; y la capacidad para regular las emociones y promover el crecimiento emocional e intelectual" (MAYER \& SALOVEY, 1997:10), en Extremera, N. y Fernández-Berrocal, P. (2004).

En principio, parece lógico pensar que los alumnos que puntuan más alto en I.E. van a tener un mayor éxito, pero en los primeros estudios anglosajones realizados a universitarios, se demostró que no existía una correlación directa en la I.E. y el rendimiento académico. Otros estudios realizados por Newson, Day y Catano (2000) realizados a estudiantes canadienses avalaron que no existía relación positiva entre la IE. Medida con el EQ-i y el rendimiento en Natalio Extremera Pacheco, Pablo Fernandez-Berrocal, (2004).

Podemos encontrar muchos estudios que realzan la necesaria valoración y potencialidad de la Inteligencia Emocional en el ámbito educativo. Un artículo sobre la importancia demostrada de la inteligencia emocional en adultos que habían tenido un fracaso anterior en el sistema educativo, y que han podido obtener un título mejorando su competencia labora, a través de un programa en el que se mejora las habilidades emocionales mediante un programa que denominaron "retratos": Pedro HernándezGuanir, José Eladio Ramos Cáceres, "Un programa de Inteligencia Emocional en la Educación de Personas Adultas", en el libro "Avances en el estudio de la Inteligencia Emocional: I Congreso Internacional de la Inteligencia Emocional”.

La mayor parte de estudios relacionando la Inteligencia Emocional con el éxito o fracaso en el sistema educativo se centra en Universitarios, en adultos y poco a poco se van incorporando los estudios con adolescentes.

Aunque, la mayoría de los estudios han sido realizados con muestras de estudiantes universitarios, cada vez más están aflorando los trabajos empíricos realizados con adolescentes (Ciarrochi, Chan y Bajgar, 2001; Fernández-Berrocal, Extremera y Ramos, 2003a; Liau, Liau, Teoh y Liau, 2003; Trinidad y Johnson, 2002). Tras revisar estas investigaciones, encontramos cuatro áreas fundamentales en las que una falta de IE provoca o facilita la aparición de problemas entre los estudiantes. De forma resumida, los problemas del contexto educativo asociados a bajos niveles de IE serían cuatro:

1. Déficit en los niveles de bienestar y ajuste psicológico del alumnado

2. Disminución en la cantidad y calidad de las relaciones interpersonales

3. Descenso del rendimiento académico

4. Aparición de conductas disruptivas y consumo de sustancias adictivas.

Por Natalio Extremera Pacheco, Pablo Fernández-Berrocal (2004)

Nosotros, en este trabajo vamos a comprobar si existe relación entre la inteligencia emocional de un alumno y las calificaciones en las áreas instrumentales de Lengua Castellana y Matemáticas en un grupo concreto de alumnos de tercero de primaria, con el fin de intentar verificar o no los estudios anteriores. $Y$ comprobar si el descenso o aumento del rendimiento académico esta relacionado con la IE.

\section{Método}

\section{Participantes}

Los sujetos participantes son alumnos de un colegio de la población de Santa Pola, que se encuentran en el nivel de tercero, grupo B. Son un total de 28 alumnos, de los cuales 
se descartan dos alumnos por tener una incorporación reciente y desconocer el idioma, con lo que le va a resultar complejo que pueda responder.

El centro se encuentra en una gran urbanización en un entorno considerado "socioeconómico medio", según la Consellería de educación. Se trata de una muestra con la diversidad típica de un grupo tan numeroso, compuesto por 11 niños y 15 niñas.

\section{Medidas}

Para medir la Inteligencia emocional hemos utilizado una adaptación realizada por mi, con el fin que los alumnos puedan comprender los ítems de cada cuestión. Ha habido ligeros cambios centrados en suavizar la complejidad del vocabulario del cuestionario original, pero de modo que siga lo más fielmente posible, la esencia del cuestionario.

Para ello, hemos tenido en cuenta el Cuestionario BarOn EQ-i YV (S) de Reuven BarOn, Ph.D. \& James D. A. Parker, Ph. D., traducido por; Carmen M. Carballo, Orlando Villegas, Ph. D. Según Ugarriza (2001) el cuestionario tiene un coeficiente de consistencia interna de .93 .

El instrumento esta compuesto por 30 ítems a los que los alumnos deben contestar según la escala Likert (1: no es verdad en mi caso, 2: un poco cierto en mi caso, 3: es verdad en mi caso, 4: muy de acuerdo en mi caso). Y nos da la información relativa a 4 aspectos de la IE: factor intrapersonal (cuestiones 18, 21, 26, 27, 29), factor interpersonal (cuestiones 17, $18,20,23,28,30$ ), factor de manejo del estrés (cuestiones17, 21, 23, 24, 27,29) y factor de adaptabilidad (cuestiones $16,19,22,24,25,28$ ), así como una puntuación total de la IE (suma de A, B, C y D).

La inteligencia Intrapersonal evalúa las habilidades relativas de autoconciencia emocional, autoestima, asertividad, auto actualización, independencia. La Inteligencia Interpersonal, comprende la empatía, las relaciones interpersonales y la responsabilidad social. La Gestión del estrés, incluye la tolerancia al estrés y el control de impulsos y la adaptabilidad, comprende las habilidades relacionadas con la solución de problemas, la comprobación de la realidad y la flexibilidad.

Los datos obtenidos se analizaran utilizando SPSS por su simplicidad y facilidad de manejo, en donde podemos extraer, tanto las medias, graficas de dispersión, coeficientes de correlación y todo lo necesario para este estudio.

\section{Procedimiento}

Se solicitó permiso a la dirección del centro para poder realizar esta prueba, y no pusieron impedimento siempre y cuanto los cuestionarios fuesen anónimos y los nombres de los alumnos no aparezca en ningún lugar público.

Los alumnos tienen una jornada continua de 9 a $14 \mathrm{~h}$, con un descanso de 25 minutos a las $11,15 \mathrm{~h}$. Otro pequeño descanso de $5 \mathrm{~min}$. A las 13,10h. Según los tutores de $3^{\circ}$, los alumnos tienen un mayor rendimiento de trabajo durante las tres primeras sesiones, antes justo del primer descanso. Por tanto aproveche un día a las 9 de la mañana y tuvimos que explicar brevemente como responder al cuestionario, pues el formato de cuestionario era desconocido para los alumnos. Tras la explicación y dirección para que sepan responder, los alumnos realizaron el cuestionario. Al final necesitamos $1 \mathrm{~h}$, entre prepararlos, explicarles y realizarlo.

\section{Análisis de datos}

El método empleado para la investigación es cuasi-experimental debido a que la selección del grupo a investigar no ha sido al azar sino por posibilidades de acceso a la muestra. Y la forma de análisis de los datos correlacional típica, ya que se pretende 
comprobar si existe relación directa de la Inteligencia emocional con las calificaciones trimestrales del los alumnos en las áreas instrumentales.

Como podemos observar en la tabla 1 (ANEXO 1), los sujetos están numerados, con su inicial del nombre y una " $M$ " si es niña, o una "V" si es niño. En la columna $3^{\mathrm{a}}$ tenemos las notas finales del área de matemáticas, con una nota media de los alumnos de 5,9. En la columna $4^{\text {a }}$, tienen una nota media de 7,3 y en la $5^{\circ}$ columna comprobamos los resultados totales obtenidos en el cuestionario, con una media de 62,4. Los parciales, se encuentran en las columnas nombradas como "A", "B"," C", "D", que hacen referencia a la escala interpersonal, intrapersonal, manejo del estrés y adaptabilidad, respectivamente.

En color rojo, los datos que están por debajo de la media y en verde los datos que están por encima de la media del grupo. He encuadrado las referencias coincidentes, es decir, notas por debajo de la media en todos los casos o superiores a la media en todos los casos.

\begin{tabular}{|c|c|c|c|c|c|c|c|}
\hline & MATEMÁTICAS & LENGUA & $\mathrm{E}$ & $\mathrm{A}$ & $\mathrm{B}$ & $\mathrm{C}$ & $\mathrm{D}$ \\
\hline \multicolumn{8}{|l|}{ MATEMÁTICAS } \\
\hline LENGUA &, $671^{* *}$ & & & & & & \\
\hline $\mathrm{E}$ &, 122 &,- 021 & & & & & \\
\hline A &, 139 &,- 155 &, $512^{* *}$ & & & & \\
\hline B &, 175 &, 261 &, $670^{* *}$ & ,387 & & & \\
\hline $\mathrm{C}$ &,- 087 &,- 233 &, $498^{* *}$ &,- 145 &,- 054 & & \\
\hline $\mathrm{D}$ &, 273 &, 341 &, $785^{* *}$ & 357 &, $638^{* *}$ &, 134 & \\
\hline Media & 5,88 & 7,27 & 62,38 & 13,46 & 19,27 & 12,31 & 16,73 \\
\hline Desviación Típica & 1,608 & 1,845 & 9,038 & 3,114 & 3,244 & 4,856 & 3,748 \\
\hline
\end{tabular}

Figura 2. Datos estadísticos. SPSS. Notas trimestrales y resultados cuestionario EQ-i (YV) de Bar-On.

\section{Resultados}

El coeficiente de correlación entre matemáticas y el total del cuestionario "E" es cercano a "o", con lo que significa que hay una correlación positiva muy baja o nula. Con lo que respecta al área de Lengua, la correlación es negativa muy baja tendente a nula (excepto con B, que aún siendo positiva es muy baja). En ambos caso es baja o nula.

La correlación entre las áreas instrumentales, Lengua y Matemáticas es altamente significativa. Del mismo modo que el Total del cuestionario "E", correlaciona positivamente y, lógicamente, con las parciales del cuestionario "A", "B", "C" y "D".

Destacar la correlación positiva dentro de los resultados parciales del cuestionario, existe una correlación alta entre intrapersonal (B) y adaptabilidad (D) 


\section{Conclusiones}

La primera conclusión a la que se puede llegar es que no existe una correlación directa entre los resultados de las notas y los resultados totales de la prueba de Inteligencia Emocional, puesto que como podemos observar, son cercanos a 0 . De este modo, se puede concluir que, en lo relativo a la cuestión de investigación, cabe reseñar que los resultados indican que no existe relación directa entre las notas de los alumnos y los resultados obtenidos con el cuestionario EQ-i (YV), al menos de un modo directo.

La correlación positiva entre las 2 áreas con alta significatividad, no nos es útil para el estudio, además de tener una lógica implícita a que se trata del mismo profesor quien imparte ambas áreas y puesto que las características de los alumnos son las determinantes de sus resultados obtenidos en un determinado entorno educativo.

Cabe destacar que entro de los resultados parciales del cuestionario, existe una correlación alta entre intrapersonal (B) y adaptabilidad (D). Obviaremos la correlación alta entre la total y las parciales por formar parte de ese total.

Por otro lado se puede observar que la media obtenida por la clase, en el cuestionario, es un poco inferior a la establecida como media en la tabla de evaluación de respuestas (ver Figura 1, en ANEXO 1). La prueba utilizada esta pensado para adolescentes, y aunque la tabla de corrección permite analizar los datos para edades como la que comprende la investigación, y aún habiendo adaptado el cuestionario no refleja las necesidades para la medición de la IE de estos alumnos.

Reseñar que aunque la hipótesis propuesta no se comprueba, y como ya hemos comentado, existen números estudios que indican la relación existente entre Inteligencia emocional y empatía (Extremera et all. 2004), entre Inteligencia emocional y competencia social como encontramos en diferentes autores, citados en Gilar Corbí et all. (2008), no se encuentran estudios exhaustivos sobre Inteligencia emocional, primaria y rendimiento académico, y en la actualidad encontramos algunos únicamente con adultos y adolescentes a los que nos hemos referido.

Las debilidades de la investigación es principalmente que la muestra es insuficiente para resultar significativa, no por ello el trabajo resulta menos interesante como base para la revisión de estudios similares, y para comprobar que relación no es directamente proporcional. Además es interesante para proponer que se realicen estudios específicos destinados a evaluar la Inteligencia Emocional en este tramo de edad.

\section{Referencias}

Albert Pla, D., Gilar Corbí, R. y Castejón Costa, J.L. Inteligencia general, Inteligencia Emocional y Habilidades sociales como predictores del rendimiento en estudiantes de Educación Secundaria Obligatoria. Investigación e innovación Educativa. En FernándezBerrocal, P., Extremera, N., Palomera, R., Ruiz-Aranda,

D., Salguero, J.M. y Cabello, R. (Coord.) (2009). Avances en el estudio de la Inteligencia Emocional: I Congreso Internacional de la Inteligencia Emocional. Santander: fundación.

Daniel Goleman, (1995). Inteligencia emocional. 
Gilar Corbí, R., Miñano Pérez, P. y Castejón Costa, J.L. (2008). Inteligencia Emocional y Empatía: su influencia en la Competencia Social en Educación Secundaría Obligatoria. SUMMA Psicológica UST, 5 (1), 21-32.

Ferragut, M., Fierro Bardají, A. (2012). Inteligencia emocional, bienestar personal y rendimiento académico en preadolescentes. Revista latinoamericana de psicología, 44 (3), pp. 95-104.

Martín Jorge, M. L. y Mora Mérida, J. A. (2004). Relación entre Inteligencia Emocional y personalidad en sus distintas concepciones teóricas. En Fernández-Berrocal, P. Et all. (2009). Avances en el estudio de la Inteligencia Emocional: I Congreso Internacional de la Inteligencia Emocional. P 91-97, Santander: fundación.

Extremera Pacheco, N. y Fernandez-Berrocal, P. (2004). El papel de la inteligencia emocional en el alumnado: evidencias empíricas. Revista Electrónica de Investigación Educativa, 6 (2). 


\section{Anexo 1}

\begin{tabular}{|c|c|c|c|c|c|c|c|c|}
\hline & MUESTRA & MATEMÁTICAS & LENGUA & BARON "E" & $\mathbf{A}$ & B & $\mathbf{C}$ & $\mathbf{D}$ \\
\hline 1 & M.M & 6,0 & 6,0 & 57 & 12 & 18 & 14 & 13 \\
\hline 2 & M.V & 9,0 & 10,0 & 65 & 14 & 20 & 16 & 15 \\
\hline 3 & P.M & 6,0 & 8,0 & 72 & 18 & 21 & 11 & 22 \\
\hline 4 & N.M & 4,0 & 7,0 & 65 & 10 & 19 & 20 & 16 \\
\hline 5 & D.V & 5,0 & 6,0 & 57 & 15 & 13 & 16 & 13 \\
\hline 6 & F.V & 4,0 & 4,0 & 67 & 18 & 24 & 6 & 19 \\
\hline 7 & J.M & 5,0 & 8,0 & 71 & 15 & 24 & 13 & 19 \\
\hline 8 & A.M & 9,0 & 10,0 & 54 & 12 & 20 & 6 & 16 \\
\hline 9 & A.M & 4,0 & 7,0 & 64 & 10 & 19 & 17 & 18 \\
\hline 10 & M.M & 9,0 & 10,0 & 72 & 16 & 23 & 10 & 23 \\
\hline 11 & L.M & 5,0 & 7,0 & 62 & 18 & 21 & 6 & 17 \\
\hline 12 & T.V & 6,0 & 9,0 & 66 & 16 & 23 & 6 & 21 \\
\hline \multicolumn{9}{|c|}{13} \\
\hline 14 & G.V & 5,0 & 6,0 & 48 & 15 & 17 & 7 & 9 \\
\hline 15 & I.V & 6,0 & 7,0 & 57 & 15 & 16 & 12 & 14 \\
\hline 16 & S.V & 7,0 & 6,0 & 55 & 12 & 16 & 13 & 14 \\
\hline 17 & X.V & 4,0 & 4,0 & 48 & 11 & 11 & 8 & 12 \\
\hline 18 & E.M & 7,0 & 8,0 & 67 & 16 & 21 & 12 & 18 \\
\hline 19 & M.M & 8,0 & 10,0 & 61 & 8 & 20 & 12 & 21 \\
\hline \multicolumn{9}{|c|}{20} \\
\hline 21 & N.V & 5,0 & 6,0 & 69 & 12 & 20 & 12 & 15 \\
\hline 22 & C.M & 6,0 & 7,0 & 73 & 14 & 17 & 19 & 23 \\
\hline 23 & E.M & 8,0 & 9,0 & 56 & 14 & 17 & 10 & 15 \\
\hline 24 & A.V & 5,0 & 7,0 & 58 & 10 & 22 & 11 & 15 \\
\hline 25 & D.V & 5,0 & 5,0 & 72 & 12 & 20 & 24 & 16 \\
\hline 26 & A.M & 5,0 & 5,0 & 65 & 14 & 20 & 16 & 15 \\
\hline 27 & A.M & 4,0 & 10,0 & 41 & 6 & 16 & 6 & 13 \\
\hline 28 & A.V & 6,0 & 7,0 & 80 & 17 & 23 & 17 & 23 \\
\hline \multicolumn{2}{|c|}{ media } & 5,9 & 7,3 & 62,4 & 13,5 & 19,3 & 12,3 & 16,7 \\
\hline \multicolumn{2}{|c|}{ Media BARON } & & & 70 & 15,5 & 20,5 & 17,5 & 18,5 \\
\hline
\end{tabular}

Figura 1. Resumen de datos 